\title{
Remote Ultrasound Palpation for Robotic Interventions Using Absolute Elastography
}

\author{
Caitlin Schneider $^{1}$, Ali Baghani ${ }^{1}$, Robert Rohling ${ }^{1,2}$, and Septimiu Salcudean ${ }^{1}$ \\ 1 Department of Electrical and Computer Engineering, \\ University of British Columbia, Vancouver, BC, Canada \\ 2 Department of Mechanical Engineering, \\ University of British Columbia, Vancouver, BC, Canada
}

\begin{abstract}
Although robotic surgery has addressed many of the challenges presented by minimally invasive surgery, haptic feedback and the lack of knowledge of tissue stiffness is an unsolved problem. This paper presents a system for finding the absolute elastic properties of tissue using a freehand ultrasound scanning technique, which utilizes the da Vinci Surgical robot and a custom 2D ultrasound transducer for intraoperative use. An external exciter creates shear waves in the tissue, and a local frequency estimation method computes the shear modulus. Results are reported for both phantom and in vivo models. This system can be extended to any 6 degree-of-freedom tracking method and any $2 \mathrm{D}$ transducer to provide real-time absolute elastic properties of tissue.
\end{abstract}

Keywords: Ultrasound, Absolute Elastography, Robotic Surgery.

\section{Introduction}

During laparoscopic procedures, surgeons face challenges such as limited vision of the surgical site and lack of dexterity and haptic feedback. In this type of surgery, the organs are only touched with the distal ends of long surgical instruments that must pass through the patient's abdominal wall. While the da Vinci Surgical System (Intuitive Surgical, Sunnyvale, CA) has overcome with some of the issues that make laparoscopic surgery difficult, including stereoscopic vision and improved tool dexterity [7, the issue of haptic feedback remains unsolved.

Ultrasound elastography has the potential to offer an alternative to providing haptic feedback by instead providing a full image of tissue stiffness and viscosity - the very properties that surgeons try to measure during manual palpation. Ultrasound imaging is relatively inexpensive, non-ionizing and real-time, making it an advantageous imaging modality for intra-operative navigation. Conventional ultrasound has been integrated previously into the da Vinci Surgical System using multiple types of ultrasound transducers [14 15].

Previous ultrasound elastography has been primarily based on strain imaging. Ultrasound strain imaging, which provides images of relative tissue deformation in response to various compression levels applied by the ultrasound transducer 
12, has also been integrated with the da Vinci Surgical System [4. That system uses the 'Read-Write' Application Programming Interface (API) to overlay a palpation motion onto the movements of the surgeon. This removes some of the user-related difficulties of creating quality strain images by moving the transducer with a known amplitude and frequency. Strain imaging can be used to determine the tumour extent and for image registration [16, but is more affected by boundary conditions.

Acoustic radiation force imaging (ARFI) has shown promising results, and been tested in vivo [10:5]. This method of imaging uses the acoustic force produced by the ultrasound transducer to create a shear wave in the tissue, whose speed is then captured through fast imaging techniques and correlation based methods. Unfortunately, this method requires high powered ultrasound machines.

This paper proposes a novel freehand absolute elastography method for the da Vinci robot. The method uses an external exciter to induce vibrations into the patient's body, while a 2D intra-operative transducer, manoeuvred by the surgeon, is used to acquire $1 \mathrm{D}$ axial vibration amplitude and phase over a volume. These vibration phasors are acquired at known 3D locations by using the da Vinci 'Read' API to determine the position and orientation of the transducer. In the paper, 'axial' will refer to the direction in the image away from the transducer face.

To the best of our knowledge, this is the first time that an absolute elastography approach with external excitation has been developed using the position and orientation of a 2D transducer for freehand sampling of a tissue volume. Contributions of this paper include the novel use of $2 \mathrm{D}$ tracked ultrasound to create 3D absolute elastograms, the use of external excitation which does not require specialized ultrasound hardware and the integration of elastography with robotic surgery.

\section{Methods}

\subsection{Elastography}

Absolute ultrasound elastography is based on the measurement of shear wave propagation in tissue in response to a mechanical excitation. The excitation can be generated by an acoustic impulse radiation force [10 or by an external vibrator [11. We use an external vibrator in our work because there are no issues related to penetration depth and tissue heating and because we can use a standard, limited power ultrasound machine. For a harmonic excitation, shear wave propagation in a homogeneous elastic solid, for small strain and linear approximations, is described in the frequency domain by the following wave equation:

$$
\rho(j \omega)^{2} \hat{\boldsymbol{u}}(x, j \omega)=\mu \nabla^{2} \hat{\boldsymbol{u}}(x, j \omega)
$$

We make the assumptions that the density of tissue $\rho$ is homogeneous and equal to that of water, although tissue density does change with tissue type and effects the wave speed slightly, this assumption is common in the elastography community. We also make the assumption that the displacement phasors $\hat{\boldsymbol{u}}(x, j \omega)$ 
A)
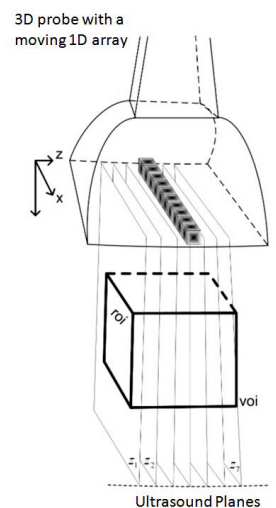

B)

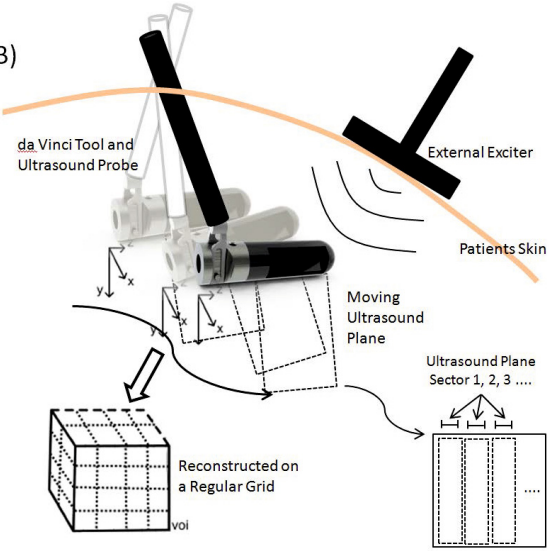

Fig. 1. A) Elastography set-up for the 3D probe method. B) Set-up for the extension to freehand scanning using a $2 \mathrm{D}$ transducer and the da Vinci robot. In both cases, sector subdivision high-frame rate imaging is applied.

generated in tissue by the vibrator have only a shear wave component. Then, if one component of the displacement phasors $\hat{\boldsymbol{u}}(x, j \omega)$ is known over a region of interest, the shear wave equation (11) can be used to find the shear modulus $\mu$. The phasors are then interpolated over a regular 3D grid covering the acquisition volume, and the distribution of shear moduli over the tissue is estimated by using a local spatial frequency estimator [9]. The estimation method is based on directional filtering of the displacement image using a set of filter banks, for which the ratio of the outputs determines the local frequency of the image.

The shear modulus provided with this system is an absolute measure of elasticity that, unlike strain imaging, is less dependent on boundary conditions. Strain is inversely proportional to elasticity under certain conditions, such as uniform stress. Changing boundaries, violates this assumption, which results in image artifacts which do not represent the elasticity of tissue, but are a result of nonuniform stress field within the tissue. The presented method however measures the local wavelength of the waves traveling in the tissue, which is dependent on the local intrinsic elasticity of the tissue, and not the boundary conditions.

With a steady state excitation, the axial displacement phasors can be estimated over a volume at a high effective frame rate using a sector-based approach [Figure 1] [1. With this method each sector is imaged several times. Because this sector is small, imaging can be completed at a frame rate 16 times higher than if the image lines were acquired in sequence, in this case, about $1.25 \mathrm{kHz}$. The image acquisition is synchronized with the external excitation such that the sectors can be reconstructed to create a single frame using time delay compensation [1].

Our system uses an external mechanical exciter (LDS Model V203, B\&K, Denmark) driven by waveforms created by a signal generator (Agilent 33220A) controlled by a standard PC. The excitation is applied using a $3.5 \mathrm{~cm}$ diameter 
circular plate. No particular positioning of the exciter with respect to the transducer is used.

\subsection{Freehand Technique}

A mechanical 3D probe has been used previously to capture a $3 \mathrm{D}$ volume of tissue displacements [Figure 1A] [2]. Such a probe mechanically sweeps a 1D crystal array to create regularly spaced ultrasound planes, but can be large and bulky. In this paper, we use a tracked $2 \mathrm{D}$ transducer to create the same type of $3 \mathrm{D}$ volume [Figure 1B].

The custom-designed transducer creates a static and repeatable transform between the da Vinci tool (Prograsp) and the ultrasound image. The transducer has 128 elements, is $28 \mathrm{~mm}$ long and is operated at 7 to $10 \mathrm{MHz}$. Image sector size was 8 lines. The tool-to-image transform was found using the single-wall phantom method implemented in Stradwin [13.

To synchronize the external exciter with the ultrasound image acquisition, the surgeon using the transducer triggers the image capture by using the clutch pedal in the robot console, an event captured by the da Vinci API. This approach has the benefit of keeping the ultrasound transducer still while imaging takes place but has the drawback that it interferes with the natural, smooth motion of ultrasound scanning. See Section 4 for methods of improving upon the natural movement limitation.

Image acquisition begins with the collection of radio frequency $(\mathrm{RF})$ data along the axial direction of the ultrasound image. The RF data is used to compute the displacement phasors $\hat{\boldsymbol{u}}(x, j \omega)$ at all depths in the image with respect to the transducer face. When a full volume of displacements phasors is captured, typically 15-30 frames, the real and imaginary parts of the axial displacement phasors (scalar values) are reconstructed into a volume on a regular grid. The volume reconstruction is performed using the PLUS software architecture [8] and uses linear interpolation between points for the real part and the imaginary part of the estimated displacement phasors. The field of view with an intra-operative transducer is small, about $30 \mathrm{~mm}$ by $40 \mathrm{~mm}$, which means the computational time required for reconstruction is minimal. The absolute elastic properties of the samples are found from the grid displacements using local frequency estimation [Section 2.1]. This final volume of elasticity can be displayed to the operating surgeon or used to create a local model for haptic feedback.

\section{$3 \quad$ Experimental Set-Up and Results}

\subsection{Elastography Phantom}

A CIRS Elastic Quality Assurance (QA) Phantom, model 049 (Computerized Imaging Reference Systems, Norfolk, VA), was used to evaluate the accuracy of the elastic properties found using this method. The soft $(6 \mathrm{kPa})$ and stiff $(54$ $\mathrm{kPa}$ ) shallow lesions were imaged against a neutral background (29 kPa). Image 

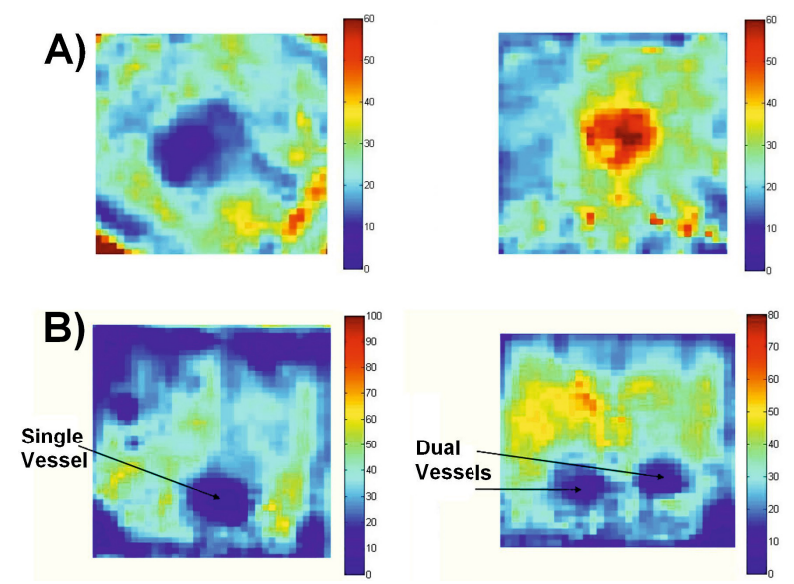

Fig. 2. A) Left: Image of $6 \mathrm{kPa}$ CIRS phantom lesion. Right: Image of the $54 \mathrm{kPa}$ CIRS phantom lesion. The colour bar shows the elasticity in kilopascals $(\mathrm{kPa})$. B) Crosssectional elastograms of the flow phantom. Left: Single vessel. Right: dual vessels after bifurcation. The colour bar shows the elasticity in kilopascals.

acquisition was performed simultaneously at four different frequencies: 180, 210, 230 and $270 \mathrm{~Hz}$.

The soft and stiff lesions were imaged at a depth of $4 \mathrm{~cm}$ and a frequency of 7 $\mathrm{MHz}$ and the resulting elastograms are shown in Figure 2A. The diameter and average elasticity were measured in two images (corresponding to the middle of the lesion) for 5 trials for each lesion. The diameters of each lesion were also measured using the caliper function of the ultrasound software [Table 1]. The results for the stiff lesion were within $6 \%$ error of the manufacturer's specifications and within $4 \%$ of the values found using magnetic resonance elastography (MRE) on the same phantom model [3. The softer lesion was stiffer than the manufacturer specified value but within $2 \%$ of the values reported with MRE. The results achieved through freehand ultrasound elastography are repeatable, with narrow standard deviations in both the diameter measurements and the elastic properties.

\subsection{Vessel/Flow Phantom}

A vessel phantom (Blue Phantom, Redmond, WA) with a single incoming vessel that branches in two halfway through the phantom was used to demonstrate the ability to highlight vessels. Because fluid does not support the transmission of shear waves, the shear wave amplitude drops significantly within the vessels. Areas within the image that show no presence of shear waves at the frequencies of excitation correspond to vessels or cysts or other fluid-filled cavities. See Section 4 for further discussion of flow imaging.

The results of these scans are shown in Figure 23. The vessels before and after the bifurcation point can be clearly seen in the elastograms. 
Table 1. Results from the CIRS QA Elastography Phantom

\begin{tabular}{|c|c|c|}
\hline & Diameter of Soft Lesion & Diameter of Stiff Lesion \\
\hline B-Mode & $8.9 \pm 0.6 \mathrm{~mm}$ & $10.8 \pm 0.2 \mathrm{~mm}$ \\
\hline Elastogram & $10.4 \pm 1.6 \mathrm{~mm}$ & $10.7 \pm 1.6 \mathrm{~mm}$ \\
\hline & & \\
\hline & Elasticity of Soft Lesion & Elasticity of Stiff Lesion \\
\hline Manufacturer Specifications & $6 \mathrm{kPa}$ & $54 \mathrm{kPa}$ \\
\hline MR Elastography [3 & $11.1 \pm 2.1 \mathrm{kPa}$ & $49.4 \pm 16.9 \mathrm{kPa}$ \\
\hline Freehand Elastography & $10.9 \pm 0.6 \mathrm{kPa}$ & $51.1 \pm 5.2 \mathrm{kPa}$ \\
\hline
\end{tabular}

\subsection{In vivo Feasibility}

In order to determine if this type of absolute elastography imaging is feasible in vivo, experiments were performed on a healthy volunteer. In this case only, we used a 3D motorized ultrasound transducer to image a healthy kidney. The purpose of this experiment was to prove that an external exciter will sufficiently transmit waves into the body to create quality elastograms. The results of the in vivo kidney scans are shown in Figure 3. The kidney is circled in both images. The outline of the kidney can be approximately delineated in the elastogram, as well as the contrasting internal collecting system.

In addition to the use with kidneys, studies have shown that B-mode and strain imaging are useful for prostatectomy [6], and the system described in this paper could easily be applied to this procedure. The surgeon would also have the option to combine information from a trans-rectal ultrasound transducer and the da Vinci controlled transducer.

\section{Discussion and Future Work}

This paper presents the method and associated results for 3D absolute elastography with a freehand scanning technique. Specifically, a small 2D intra-operative probe allows robotic laparoscopic surgeons access to the elastic values of tissue. The results with freehand imaging are comparable to both those achieved with a mechanical 3D probe [2] and within 5\% of the results achieved with magnetic resonance imaging techniques 3 . We have demonstrated this system's accuracy in a phantom model and feasibility in an in vivo setting.

The method is currently implemented using the da Vinci robotic system but can easily be extended to any 2D probe and any tracking system, such as electromagnetic or optical tracking systems. This would allow absolute elasticity values to be found in nearly every clinical setting. We have used the da Vinci robot as an initial platform for integration for several reasons; the robotic environment has the most room for improvement with regards to haptic feedback, and at the same time provides a stable and accurate platform for transducer tracking. The quality of the elastogram is dependent on the tracking error, which in turn depends on the tracker involved. Generally speaking though, if the planes are dense, a better elastogram is obtained, because of the higher signal to noise ratio due to averaging. 

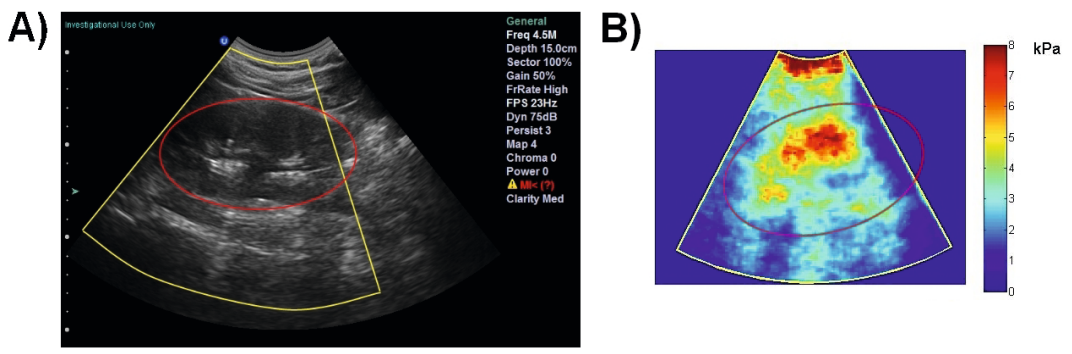

Fig. 3. Images of a healthy kidney circled in red. Left: B-Mode image. Right: Elastogram.

One current limitation of the system is synchronization of the imaging and the external exciter. This is currently addressed by using the clutch pedal in the surgeon's console to trigger imaging at a specified phase of the exciter. With accurate time stamps on the image acquisition and knowledge of the exciter phase, each sector or image could be placed in a temporal and spatial location within the end volume. This information would allow the surgeon to move the transducer in the same smooth manner that is used while scanning with only B-mode.

As mentioned above, elastic imaging could be used to visualize vessels as well as elastic properties. Shear waves do not propagate in fluid causing a marked decrease in the wave amplitude at the frequencies of the external exciter. Interestingly, the methods that are used to track the tissue motion are the same techniques that are used to create traditional Doppler images that measure the flow velocity in vessels [11. Given that the decrease in amplitude can be visualized clearly, it is possible to localize these areas and then use Doppler techniques to measure the flow velocity. With this cascade of image processing, it would be possible to automatically locate the vessels within an image and optimally position the gate for Doppler imaging. Surgeons would also be able to image both elastic properties and flow at the same time. Additionally, because the elastography algorithms are similar in multiple modalities, it is possible to register absolute ultrasound elastograms directly to other elastic imaging modalities, in particular pre-operative MRE images. Because MRE and US elastograthy measure the same quantity, the registration may become more straight forward, considerably simplifying a generally difficult problem.

In this paper, we present an absolute elastography method for a tracked 2D ultrasound transducer. Absolute elastography methods provide new quantitative information over the previous strain imaging methods. Our methods are user and exciter independent and less affected by boundary conditions. These qualities make it easy to use and possibly more repeatable than strain imaging. We implemented this system on the da Vinci in order to address its lack of haptic feedback but this method can be extended to any 2D transducer and tracking system. 


\section{References}

1. Baghani, A., Brant, A., Salcudean, S., Rohling, R.: A high-frame-rate ultrasound system for the study of tissue motions. IEEE Ultrasonics, Ferroelectrics and Frequency Control 57(7), 1535-1547 (2010)

2. Baghani, A., Eskandari, H., Wang, W., Da Costa, D., Nabil, N., Sahebjavaher, R., Salcudean, S., Rohling, R.: Real-time quantitative elasticity imaging of deep tissue using free-hand conventional ultrasound. In: MICCAI: ACCEPTED (2012)

3. Baghani, A., Salcudean, S., Honarvar, M., Sahebjavaher, R., Rohling, R., Sinkus, R.: Travelling wave expansion: A model fitting approach to the inverse problem of elasticity reconstruction. IEEE Medical Imaging (99), 1 (2011)

4. Billings, S., Deshmukh, N., Kang, H., Taylor, R., Boctor, E.: System for robotassisted real-time laparoscopic ultrasound elastography. In: SPIE Medical Imaging (2012)

5. Evans, A., Whelehan, P., Thomson, K., McLean, D., Brauer, K., Purdie, C., Jordan, L., Baker, L., Thompson, A.: Quantitative shear wave ultrasound elastography: initial experience in solid breast masses. Breast Cancer Res. 12(6) (2010)

6. Han, M., Kim, C., Mozer, P., Schafer, F., Badaan, S., Vigaru, B., Tseng, K., Petrisor, D., Trock, B., Stoianovici, D.: Tandem-robot assisted laparoscopic radical prostatectomy to improve the neurovascular bundle visualization: A feasibility study. Urology (2010)

7. Hubens, G., Coveliers, H., Balliu, L., Ruppert, M., Vaneerdeweg, W.: A performance study comparing manual and robotically assisted laparoscopic surgery using the da Vinci system. Surgical Endoscopy 17(10), 1595-1599 (2003)

8. Lasso, A., Heffter, T., Pinter, C., Ungi, T., Chen, T.K., Boucharin, A., Fichtinger, G.: Plus: An open-source toolkit for developing ultrasound-guided intervention systems. In: 4th Image Guided Therapy Workshop, vol. 4, p. 103 (2011)

9. Manduca, A., Muthupillai, R., Rossman, P., Greenleaf, J., Ehman, R.: Local wavelength estimation for magnetic resonance elastography. In: Int. Conf. on Image Processing, vol. 3, pp. 527-530. IEEE (1996)

10. Nightingale, K., Soo, M., Nightingale, R., Trahey, G.: Acoustic radiation force impulse imaging: in vivo demonstration of clinical feasibility. Ultrasound in Medicine \& Biology 28(2), 227-235 (2002)

11. Ophir, J., Alam, S., Garra, B., Kallel, F., Konofagou, E., Krouskop, T., Varghese, T.: Elastography: ultrasonic estimation and imaging of the elastic properties of tissues. Journal of Engineering in Medicine 213(3), 203 (1999)

12. Ophir, J., Cespedes, I., Ponnekanti, H., Yazdi, Y., Li, X.: Elastography: a quantitative method for imaging the elasticity of biological tissues. Ultrasonic Imaging 13(2), 111-134 (1991)

13. Prager, R., Rohling, R., Gee, A., Berman, L.: Rapid calibration for 3-D freehand ultrasound. Ultrasound in Medicine \& Biology 24(6), 855-869 (1998)

14. Schneider, C.M., Dachs II, G.W., Hasser, C.J., Choti, M.A., DiMaio, S.P., Taylor, R.H.: Robot-Assisted Laparoscopic Ultrasound. In: Navab, N., Jannin, P. (eds.) IPCAI 2010. LNCS, vol. 6135, pp. 67-80. Springer, Heidelberg (2010)

15. Schneider, C., Guerrero, J., Nguan, C., Rohling, R., Salcudean, S.: Intra-operative "Pick-Up" Ultrasound for Robot Assisted Surgery with Vessel Extraction and Registration: A Feasibility Study. In: Taylor, R.H., Yang, G.-Z. (eds.) IPCAI 2011. LNCS, vol. 6689, pp. 122-132. Springer, Heidelberg (2011)

16. Stolka, P., Keil, M., Sakas, G., McVeigh, E., Allaf, M., Taylor, R., Boctor, E.: A 3D-elastography-guided system for laparoscopic partial nephrectomies. In: SPIE Medical Imaging (2010) 